\title{
Topological model for a class of complex Hénon mappings
}

\author{
Sylvain Bonnot
}

\begin{abstract}
In order to describe the dynamics of the complex Hénon map $H_{a, c}:\left(\begin{array}{l}x \\ y\end{array}\right) \mapsto\left(\begin{array}{c}P_{c}(x)-a y \\ x\end{array}\right)$, where $P_{c}: z \mapsto z^{2}+c$ has an attractive fixed point, we build a global topological model $(g, Y)$. In this model $Y$ is the complement in $\mathbb{R}^{4}$ of a cone over a solenoid lying in the unit 3-sphere, and $g: Y \rightarrow Y$ is a map given in spherical coordinates by $g(r, \theta)=\left(r^{2}, \sigma(\theta)\right)$, where $\sigma$ is a solenoidal map of degree two. Then we prove the existence of a constant $\varepsilon>0$ such that any Hénon map $H_{a, c}$ with $0<|a|<\varepsilon$ is conjugate to our model $(g, Y)$.
\end{abstract}

Mathematics Subject Classification (2000). Primary 32H50; Secondary 37F99.

Keywords. Complex Hénon mappings, solenoidal map, topological model.

\section{Introduction}

Holomorphic dynamics in one complex variable has now reached a level of maturity, mainly through the use of the quasi-conformal maps. Since these methods are not available in the several variables setting, our understanding in this situation has not the same depth. In particular there is a need for global models explaining the topology of a given dynamical system. The main purpose of this paper is to provide such a global model, conjectured by J. H. Hubbard in 1986, for complex Hénon mappings given by

$$
H_{a, c}:(x, y) \mapsto\left(x^{2}+c-a y, x\right),
$$

where the jacobian $a$ is small and $c$ belongs to the main cardioid of the Mandelbrot set. Before we can actually state the main theorem, we will recall some simple definitions about complex Hénon mappings and then describe the topological model.

1.1. Complex Hénon mappings. When $a, c$ belong to $\mathbb{C}$, a complex Hénon map of degree two is usually defined by

$$
\begin{aligned}
F_{a, c}: & \mathbb{C}^{2} \longrightarrow \mathbb{C}^{2} \\
\left(\begin{array}{l}
x \\
y
\end{array}\right) & \longmapsto\left(\begin{array}{c}
x^{2}+c-a y \\
x
\end{array}\right) .
\end{aligned}
$$


Note that $F_{a, c}$ is a biholomorphism with constant jacobian equal to $a$. Since we are essentially interested in the situations where $a$ is small, we prefer to define our Hénon maps as Fornæss and Sibony did in [10]:

$$
\begin{aligned}
H_{a, c}: & \mathbb{C}^{2} \longrightarrow \mathbb{C}^{2} \\
\left(\begin{array}{l}
x \\
y
\end{array}\right) & \longmapsto\left(\begin{array}{c}
x^{2}+c+a y \\
a x
\end{array}\right) .
\end{aligned}
$$

It is easy to see that $H_{a, c}$ is conjugate to $F_{-a^{2}, c}$ by the linear map $\left(\begin{array}{l}x \\ y\end{array}\right) \mapsto\left(\begin{array}{c}x \\ a y\end{array}\right)$.

Following Hubbard we can introduce invariant subsets of $\mathbb{C}^{2}$ :

$$
K^{+}=\left\{\left(\begin{array}{l}
x \\
y
\end{array}\right) \mid\left\|H_{a, c}^{\circ n}\left(\begin{array}{l}
x \\
y
\end{array}\right)\right\|_{n \in \mathbb{N}} \text { is bounded }\right\},
$$

and $U^{+}=\mathbb{C}^{2}-K^{+}, J^{+}=\partial K^{+}$. We can also define the corresponding sets $K^{-}$, $U^{-}, J^{-}$for backward iteration.

Small perturbations of polynomials. When $a$ is small, we can view $H_{a, c}$ as a small perturbation of the quadratic polynomial $P_{c}: z \mapsto z^{2}+c$. In this article we will restrict us to the polynomials $P_{c}$ that have an attractive fixed point. This amounts to pick a parameter $c$ in the main cardioid $\mathcal{C}$ of the Mandelbrot set. Let us recall that the Julia set $J_{c}$ of the polynomial $P_{c}$ is the boundary of the set of non-escaping points. In the case where $c$ is in the main cardioid $\mathcal{C}$ it is well known that $J_{c}$ is a quasi-circle.

When $a$ is small enough, the map $H_{a, c}$ itself has an attractive fixed point $p$ whose basin of attraction is written $W^{s}(p)$. In [10] Fornæss and Sibony prove the existence of a partition

$$
\mathbb{C}^{2}=W^{s}(p) \cup J^{+} \cup U^{+} .
$$

In our setting, $H_{a, c}$ is more convenient than $F_{a, c}$ because when $a=0$, then $F_{a, c}$ degenerates into a simpler one-dimensional map $\left(\begin{array}{l}x \\ y\end{array}\right) \mapsto\left(\begin{array}{c}P_{c}(x) \\ 0\end{array}\right)$.

1.2. Topological model. Here we describe our topological model.

The space of the model. In $\mathbb{R}^{4}$, with its polar coordinates $(r, \theta)$ in $\mathbb{R}^{+} \times \mathbb{S}^{3}$, we know that the unit sphere $\mathbb{S}^{3}$ is made of two solid tori $\mathbb{T}_{0}$ and $\mathbb{T}_{1}$ glued along their boundaries. After a convenient rescaling, let us assume that $\mathbb{T}_{0}=\mathbb{S}^{1} \times \mathbb{D}$ where $\mathbb{S}^{1}$ is the unit circle and $\mathbb{D}$ the closed unit disc in $\mathbb{C}$. Then a map $f: \mathbb{T}_{0} \rightarrow \mathbb{T}_{0}$ is solenoidal of degree $m$ if it is conjugate to a map

$$
\sigma_{m, k}:(\zeta, z) \mapsto\left(\zeta^{m}, \frac{1}{2} \zeta+\varepsilon z \zeta^{k-m+1}\right),
$$

where $\varepsilon$ is small enough, so that the map is injective.

In [15], it is proved that for a fixed degree $m, \sigma_{m, 0}$ is the only map that can be extended to a homeomorphism $\tilde{\sigma}_{m, 0}: \mathbb{S}^{3} \rightarrow \mathbb{S}^{3}$. Moreover, then $\tilde{\sigma}_{m, 0}^{-1}: \mathbb{T}_{1} \rightarrow \mathbb{T}_{1}$ is 
also a solenoidal map of same degree. Let us write $\sigma$ the extension to the 3-sphere of the solenoidal map of degree two given by

$$
\sigma_{2,0}:(\zeta, z) \mapsto\left(\zeta^{2}, \frac{1}{2} \zeta+\varepsilon \frac{z}{\zeta}\right)
$$

Then we can consider

$$
\Sigma^{+}=\bigcap_{n \geqslant 0} \sigma^{n}\left(\mathbb{T}_{0}\right) \quad \text { and } \quad \Sigma^{-}=\bigcap_{n \geqslant 0} \sigma^{-n}\left(\mathbb{T}_{1}\right),
$$

the two invariant solenoids obtained by forward and backward iterations. In addition we define

$$
\operatorname{cone}\left(\Sigma^{-}\right)=\left\{(r, \theta) \mid r \geqslant 1, \theta \in \Sigma^{-}\right\} .
$$

Then the space $Y$ of our model is defined by $Y=\mathbb{R}^{4}-\operatorname{cone}\left(\Sigma^{-}\right)$.

The map of the model. The map $g$ in the model is given in polar coordinates by $g(r, \theta)=\left(r^{2}, \sigma(\theta)\right)$. It is a well-defined map from $Y$ to itself. We call $(g, Y)$ the model of the Hénon map.

1.3. Conjugacy theorem. We are now in position to state our main theorem:

Theorem 1 (Main theorem). For any $c$ in the main cardioid $\mathcal{C}$ of the Mandelbrot set, there exists an $\varepsilon>0$ such that: for any $a \in \mathbb{C}$ satisfying $0<|a|<\varepsilon$, there exists $a$ homeomorphism $h: \mathbb{C}^{2} \rightarrow Y$ which conjugates $H_{a, c}$ to $g: Y \rightarrow Y$.

\section{Remark 1.}

- The same theorem is true when we replace $H_{a, c}$ by the more common normalization $F_{a, c}:\left(\begin{array}{l}x \\ y\end{array}\right) \mapsto\left(\begin{array}{c}x^{2}+c-a y \\ x\end{array}\right)$.

- In the model the partition of $\mathbb{C}^{2}$ corresponds to the subsets $r \in[0,1[, r=1$, and $r>1$.

Strategy of the proof. We will find good coordinates in $U^{+}, J^{+}$, and $W^{s}(p)$. But then we need to make sure that these different systems of coordinates can be glued together in a consistent way and this is by far the most difficult part of the proof. Here is the reason: in order to extend the coordinates found in $J^{+}$we have to build a tubular neighbourhood of this set that overlaps both $U^{+}$and $W^{s}(p)$. But note that $J^{+}$is an extremely complicated three dimensional object with a fractal boundary. In particular we cannot use any usual method of construction of tubular neighbourhoods. A whole section will be devoted to the solution of this problem. 
Acknowledgments. I am very grateful to John H. Hubbard for being a source of inspiration over the years. I am also grateful to the Mathematics Department of Cornell University and the Université de Provence for their hospitality. I finished this paper under a fellowship from the French École Polytechnique, that I thank warmly for its support.

\section{Conjugacy in $J^{+}$}

The conjugacy in $J^{+}$has already been established by Hubbard and Oberste-Vorth in [16]. Here is a useful picture that the reader should keep in mind: when the jacobian $a$ becomes zero, $H_{a, c}$ degenerates into $\left(\begin{array}{l}x \\ y\end{array}\right) \mapsto\left(\begin{array}{c}P_{c}(x) \\ 0\end{array}\right)$, and for this degenerate map if we still define $J^{+}=\partial K^{+}$then we get $J^{+}=J_{c} \times \mathbb{C}$. And now it becomes clear that for any $R>0, J_{R}^{+}=J^{+} \cap\{|y| \leqslant R\}$ is a solid torus. The main step of the proof given by Hubbard and Oberste-Vorth is the following.

Theorem 2 (Conjugacy in $J_{R}^{+}$). For any $c \in \mathcal{C}$ and any $R>0$, there exists an $\varepsilon>0$ such that if $0<|a|<\varepsilon$, there exists a homeomorphism

$$
\Lambda: J^{+} \cap\{|y| \leqslant R\} \rightarrow \mathbb{T}
$$

which conjugates $H_{a, c}$ to $\sigma$.

2.1. Proof of the theorem. This is proved in [16] for the map $F_{a, c}$ instead of $H_{a, c}$. In order to prove it we need first:

Proposition 1. For any $c \in \mathcal{C}$ and any $R>0$, there exists an $\varepsilon>0$ such that if $0<|a|<\varepsilon$, we have:

(1) there exists $\alpha>0, R^{\prime}>0$ such that $J_{c} \subset \mathbb{D}_{R^{\prime}}$ and that the map

$$
\begin{aligned}
f_{P_{c}, \alpha, R^{\prime}}: J_{c} \times \mathbb{D}_{R^{\prime}} & \longrightarrow J_{c} \times \mathbb{C} \\
(\zeta, z) & \longmapsto\left(\zeta^{2}+c, \zeta+\alpha \frac{z}{2 \zeta}\right),
\end{aligned}
$$

is open, injective and maps $\left(J_{c} \times \mathbb{D}_{R^{\prime}}\right)$ into itself;

(2) there exists a homeomorphism

$$
\Psi: J^{+} \cap\{|y| \leqslant R\} \rightarrow J_{c} \times \mathbb{D}_{R^{\prime}}
$$

conjugating $H_{a, c}$ to $f_{P_{c}, \alpha, R^{\prime}}$.

Proof. (1) This is exactly lemma 1.2 of [16]. There, the two authors show that $f_{P_{c}, \alpha_{1}, R_{1}}$ and $f_{P_{c}, \alpha_{2}, R_{2}}$ are conjugate as soon as $\alpha_{1}, \alpha_{2}$ are small enough and $R_{1}, R_{2}$ are big enough. Therefore we are allowed to write $f_{P_{c}}$ only.

(2) In [16] we also have: 
Vol. 81 (2006)

Topological model for a class of complex Hénon mappings

Lemma 1. For a fixed $c \in \mathcal{C}$, there exists an annulus $A(c)$ around $J_{c}$, a real $R>0$ and a neighbourhood $N_{\delta}$ of the parabola $x \mapsto\left(\begin{array}{c}x^{2}+c \\ a x\end{array}\right)$ defined by

$$
N_{\delta}=\left\{\left(\begin{array}{l}
x \\
y
\end{array}\right) \in \mathbb{C}^{2}|| P_{c}(y / a)-x \mid<\delta\right\}
$$

satisfying:

- if $V^{\prime}=H_{a, c}\left(A(c) \times \mathbb{D}_{R}\right) \cap \operatorname{pr}_{1}^{-1}(A(c))$ and $W=V^{\prime} \cap H_{a, c}^{\circ-1}\left(V^{\prime}\right)$ then $Y=$ $J^{+} \cap W$ is homeomorphic to a solid torus;

- $H_{a, c}$ maps this solid torus into itself and is conjugate to $f_{P_{c}}$.

Proof of the lemma. This is proposition 6.4 of [16], with the little change induced by the normalization we use for our Hénon map.

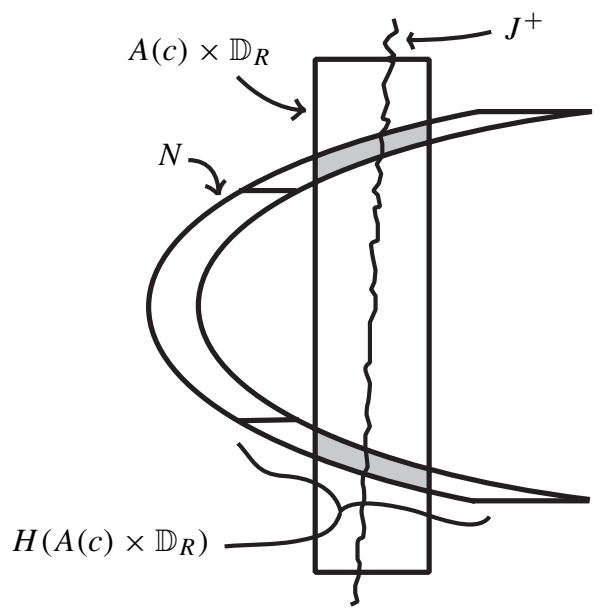

Figure 1. Conjugacy in $J_{R}^{+}$.

The construction made in [16] has the property that $W \cap J^{+}=H_{a, c}\left(J_{R}^{+}\right)$, where

$$
J_{R}^{+}=J^{+} \cap\{|y|<R\} .
$$

But now $f_{P_{c}}$ is conjugate to $\sigma_{0}$ because in [15], Theorem 3.11 it is proved that the conjugacy class of any map from the solid torus into itself that exhibits appropriate conditions of expansion and contraction depends only on its homotopy class.

So now we know the existence of homeomorphisms $h, h^{\prime}$ such that the following 
diagram commutes:

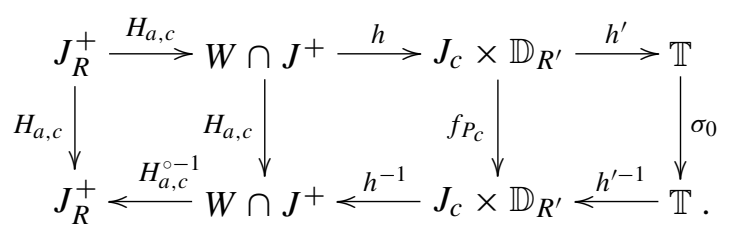

This ends the proof of the proposition and of the theorem.

Remark 2. Since Hubbard and Oberste-Vorth use $F_{a, c}$ instead, it is the parabola $x \mapsto$ $\left(\begin{array}{c}x^{2}+c \\ x\end{array}\right)$ that is fixed by the degenerate mapping $F_{0, c}$. This explains the intervention of the neighbourhood $N_{\delta}$ of the parabola. Whereas in the case of $H_{a, c}$ it is the line $\{y=0\}$ that is invariant.

2.2. Topology of $\boldsymbol{J}^{+}$. We recall some results from [16].

Inductive limits. Let $X$ be a space with a map $f: X \rightarrow X$, then the inductive limit $\check{X}_{f}=\underline{\lim }(X, f)$ is given by $\underset{\lim }{\longrightarrow}(X, f)=X \times \mathbb{N} / \sim$, where $\sim$ is defined by $(x, n) \sim(f(x), n+1)$. This limit comes with a natural map: $\check{f}: \check{X}_{f} \rightarrow \check{X}_{f}$ induced by

$$
\check{f}:(x, n) \mapsto(f(x), n) \sim(x, n-1) .
$$

This applies to $J_{c} \times \mathbb{D}_{R^{\prime}}$ together with $f_{P_{c}, \alpha, R^{\prime}}$ for small enough $\alpha$ and big enough $R^{\prime}$. Let us write then

$$
\check{\mathbb{C}}_{P_{c}}=\underline{\lim }\left(J_{c} \times \mathbb{D}_{R^{\prime}}, f_{P_{c}, \alpha, R^{\prime}}\right)
$$

Proposition 7.5 of [16] explains the topology of $J^{+}$:

Proposition 2. Let $p$ be a polynomial with an attractive fixed point attracting all the critical points of $p$, then $\check{\mathbb{C}}_{p}$ is a 3-sphere with a solenoid removed and $\check{p}$ is conjugate to $\sigma_{d, 0}:(\zeta, z) \mapsto\left(\zeta^{d}, \zeta+\varepsilon z \zeta^{1-d}\right)$.

This applies because $P_{c}$ has a single critical point and that it is in the basin of attraction of the attractive fixed point.

Going back to the model. The part in the model corresponding to $\mathrm{J}^{+}$is the unit 3-sphere with $\Sigma^{-}$removed. In order to extend this conjugacy, we will now build a tubular neighbourhood of $J_{R}^{+}$. As announced previously, this is the most difficult part of the theorem. 


\section{Tubular neighbourhood of $J_{R}^{+}$}

Let us precise the definition of a tubular neighbourhood of $J_{R}^{+}$:

Definition 1 (Tubular neighbourhood). We call tubular neighbourhood of $J_{R}^{+}$, the data $(W, h)$ satisfying:

- $W$ is a compact set such that $W \cap J^{+}=J_{R}^{+}$,

- $h$ is a homeomorphism of $W$ into a set $V$ in $\mathbb{C}^{2}$ such that

$$
V \cap \mathbb{S}^{1} \times \mathbb{C}=h\left(J_{R}^{+}\right) .
$$

Our main purpose in this section is this theorem:

Theorem 3 (Conjugacy in $W$ ). For any $c \in \mathcal{C}$ and any $R>0$, there exists $\varepsilon>0$ such that, for any a satisfying $0<|a|<\varepsilon$, there exists a tubular neighbourhood $(W, \Theta)$ of $J_{R}^{+}$such that

- $\Theta(W)=[1 / 2,2] \times \mathbb{T} \subset \mathbb{R}^{4}$;

- $\Theta$ conjugates $H_{a, c}$ to the map $g$ of the model.

3.1. Thickening of $J_{\boldsymbol{R}}^{+}$. Remember that $J_{R}^{+}$looks like $J_{c} \times \mathbb{D}_{R}$ when $|a|$ is small, so a tubular neighbourhood should look like the product of an annulus around $J_{c}$ with a vertical disk.

Proposition 3 (Tubular neighbourhood, without dynamics). For any $R>0$, any $c \in \mathcal{C}$ there exists $\varepsilon>0$ and an annulus $A(c)$ around $J_{c}$ bounded by two curves $\gamma_{0}$, $\gamma_{1}$ such that:

(1) the bounded component of $\mathbb{C}-\left(\gamma_{0}\right)$ is a topological disk $\mathbb{D}(c)$ such that, for any a with $0 \leqslant|a|<\varepsilon$, the bidisk $B=\overline{\mathbb{D}(c)} \times \mathbb{D}_{R}$ is inside the basin of attraction of the attractive fixed point;

(2) the points 0 and c are not in $A(c)$;

(3) there exists a map $\Phi: \mathbb{D}_{\varepsilon} \times \mathbb{D}_{R} \times A(c) \rightarrow \mathbb{C}^{2}$ such that:

- for any $a \in \mathbb{D}_{\varepsilon}$, the restriction of $\Phi$ to $\{a\} \times \mathbb{D}_{R} \times A(c)$ is injective,

- if $\left(x^{\prime}, y^{\prime}\right)=\Phi(a, y, x)$, then $y=y^{\prime}$,

- for all $a \in \mathbb{D}_{\varepsilon}, \Phi\left(\{a\} \times \mathbb{D}_{R} \times J_{c}\right)=J_{R}^{+}(a, c)$ (" $\Phi$ straightens $J^{+}$”),

- for fixed $x, \Phi$ is holomorphic with respect to the other variables.

3.2. Proof of the proposition about the thickening. The first point is nothing else than lemma 3.10 of [10]. For the second one the idea is to fill in a neighbourhood of $J_{R}^{+}$by solid tori made of almost vertical analytic disks. Since it is easier to do this in $U^{+}$we will split the proof. 
3.2.1. First half: $\boldsymbol{W} \cap \boldsymbol{K}^{+}$. We choose the curve $\gamma_{0}$ so that it bounds a topological disk enclosing the critical point 0 and the critical value $c$ of $P_{c}$. In addition, we take another curve $\gamma_{1}$ around $K_{c}$ : let us take for example a level set of the Green function of $K_{c}$.

These two curves bound an annulus $A(c)$ and let us define $\mathcal{U}:=A(c) \times\{|y|<R\}$. The dynamics of $P_{c}$ in the annulus $A(c)$ is described by this lemma (see theorem 5.1 of [5] for a proof):

Lemma 2 (Conjugacy with $z \mapsto z^{2}$ in an annulus). There exists a homeomorphism f satisfying:

- $f$ maps $A(c)$ onto the annulus $A=\left\{\frac{1}{2}<|z|<2\right\}$,

- $f$ maps $J_{c}$ on $\mathbb{S}^{1}$,

- $f$ conjugates $P_{c}$ to $z \mapsto z^{2}$.

At this point two constructions are possible:

(1) We can pull back successively the annulus $A(c)$ by $P_{c}$. By the previous lemma we obtain a decreasing sequence of nested annuli converging to $J_{c}$ and bounded by the curves $\gamma_{n}^{(0)}$ and $\gamma_{n}^{(1)}$. Let us write $X=J_{c} \cup \bigcup_{n} \gamma_{n}^{(0)} \cup \bigcup_{n} \gamma_{n}^{(1)}$.

(2) We can also consider the successive images of $U=A(c) \times\{|y|<R\}$ by $H_{a, c}^{-1}$ and intersect with $\{|y|<R\}$; we will write

$$
\mathcal{U}_{n}=\left\{(x, y) \in \mathcal{U} ; H_{a, c}^{\circ n}(x, y) \in \mathcal{U}\right\} .
$$

Next we set

$$
Y=J_{R}^{+}(a, c) \cup \bigcup_{n \geqslant 0}\left(\partial u_{n} \cap\{|y|<R\}\right) .
$$

Our purpose here is to give a precise meaning to the following idea: for small nonzero $a, Y$ is a small perturbation of $X \times \mathbb{D}_{R}$.

The next three lemmas proved by Fornæss and Sibony ([10], lemma 3.14, 3.15 and 3.16 ) precise how the $U_{n}$ look like.

Lemma 3 (Boundary of $\mathcal{U}_{n}$ ). The boundary in $\{|y|<R\}$ of each $\mathcal{U}_{n}$ is $\mathbb{R}$-analytic, $\partial U_{n}$ is almost vertical, and $U_{n}$ is foliated by almost vertical analytic disks given by $x=\phi(y),|y|<R$, and such that $H_{1}^{\circ n}(\phi(y), y)$ is constant for $|y|<R$.

Lemma 4 (Horizontal slices of $U_{n}$ ). For fixed $y_{0},\left|y_{0}\right|<R$ we set

$$
u_{n, y_{0}}=\left\{x ;\left(x, y_{0}\right) \in \mathcal{U}_{n}\right\} .
$$

Then $U_{n, y_{0}}$ is a connected domain with an $\mathbb{R}$-analytic boundary. The number of holes in $U_{n, y_{0}}$ does not depend on $y_{0}$ and is equal to one. If $\gamma^{n}$ is the exterior curve and $\gamma_{-n}$ the interior one then the sequence of annuli bounded by these curves is decreasing. 
Lemma 5 (The $U_{n}$ accumulate on $J_{R}^{+}$). For fixed $y_{0}$ such that $\left|y_{0}\right|<R$,

$$
\bigcap_{n \geqslant 0} u_{n, y_{0}}=J_{y_{0}}^{+}
$$

is a connected set with empty interior.

According to the first of the three lemmas, $\partial \mathcal{U}_{n}(a, c)$ is foliated by graphs $x=$ $\phi_{n}(y, a, c, s)$, where s belongs to $\partial U_{n}(a, c) \cap\{y=0\}$, and every $\phi_{n}$ is an analytic function of $(y, a, c)$. Using Hurwitz's lemma, Fornæss and Sibony obtained the next result:

Lemma 6 (Foliation of $J_{R}^{+}$). Through each point of $J_{R}^{+}=\bigcap_{n \geqslant 0} u_{n}$, there is a unique leaf in $J^{+}$given by $x=\phi(y),|y|<R$, where $\phi$ is holomorphic. Moreover, $\left|\phi^{\prime}(y)\right|$ is arbitrarily small when a tends to zero.

Foliation of $u_{0}-u_{1}$. When we take the union of the almost vertical analytic disks foliating $Y$ and we slice by the complex line $\left(y=y_{0}\right)$ we obtain a holomorphic motion of $Y \cap\{y=0\}$ parametrized by $\left(a, y_{0}\right)$. We can then apply Bers-Royden's extension theorem (see the appendix) to extend the motion to the ring bounded by $\gamma^{(0)}$ and $P_{c}^{-1}\left(\gamma^{(0)}\right)=\gamma_{1}^{(0)}$. Then we can pull-back the foliation of $\mathcal{U}-\mathcal{U}_{1}$ by $H_{a, c}$ in order to obtain a foliation preserved by the dynamics.

Lemma 7. When a tends to zero, the analytic disks foliating $u-\mathcal{U}_{1}$ become more and more vertical.

Proof. This is a direct application of our variant of Bers-Royden's theorem that we prove in the appendix.

Foliation of $u_{n}-u_{n+1}$. Since we know that the $\left(u_{n}\right)_{n \geqslant 1}$ cut every horizontal slice $\left\{y=y_{0}\right\}$ into a decreasing sequence of annuli, we can write $u=J_{R}^{+} \cup \bigcup_{n \geqslant 0}\left(U_{n}-\right.$ $\left.u_{n+1}\right)$. So we just have to fill in the next "shells" $u_{n}-u_{n+1}$ by almost vertical analytic disks. In order to do this we use a graph-transform method. The following lemma tells us that $\mathbb{D} H^{-1}$ maps an almost vertical vector in $T W$ on an almost vertical vector.

Lemma 8 (Invariant cones). For any $K>1$ there exists $\varepsilon>0$ such that for any a, $0<|a|<\varepsilon$, the following holds: if we set

$$
C_{K}\left(\begin{array}{l}
x \\
y
\end{array}\right)=\left\{\left(\begin{array}{l}
u \\
v
\end{array}\right) \in T_{(x, y)} \mathbb{C}^{2}|| u\left|\leqslant \frac{1}{K}\right| v \mid\right\},
$$

then for any $\left(\begin{array}{l}x \\ y\end{array}\right) \in W$ such that $H_{a, c}\left(\begin{array}{l}x \\ y\end{array}\right) \in W$, we have

$$
\left(\mathbb{D} H_{a, c}\left(\begin{array}{l}
x \\
y
\end{array}\right)\right)^{-1}\left(C_{K}\left(H_{a, c}\left(\begin{array}{l}
x \\
y
\end{array}\right)\right)\right) \subset C_{K}\left(\begin{array}{l}
x \\
y
\end{array}\right) .
$$


Proof. For fixed $c$, we know that $A(c)$ does not contain the origin and that there exists $r>0$ independent of $c$ such that for all $\left(\begin{array}{l}x \\ y\end{array}\right) \in W$, we have $|x| \geqslant r$.

Now

$$
\left(\mathbb{D} H_{a, c}\left(\begin{array}{l}
x \\
y
\end{array}\right)\right)^{-1}\left(\begin{array}{l}
u \\
v
\end{array}\right)=\left(\begin{array}{cc}
0 & 1 / a \\
1 / a & \frac{-2 x}{a^{2}}
\end{array}\right)\left(\begin{array}{l}
u \\
v
\end{array}\right)=\left(\begin{array}{c}
\frac{v}{a} \\
\frac{u}{a}-\frac{2 x v}{a^{2}}
\end{array}\right) .
$$

But we know that $|u| \leqslant \frac{1}{K}|v|$ and then $\left|\frac{u}{a}-\frac{2 x v}{a^{2}}\right| \geqslant\left(\frac{2 r}{|a|}-\frac{1}{K}\right) \cdot\left|\frac{v}{a}\right|$. Thus if we choose $|a| \leqslant \varepsilon=\frac{2 r}{K+\frac{1}{K}}$, then $\left(\frac{2 r}{|a|}-\frac{1}{K}\right) \cdot\left|\frac{v}{a}\right| \geqslant K\left|\frac{v}{a}\right|$.

The next proposition amounts to say that an almost vertical analytic disk in $W$ is mapped into a thin parabola by $H^{-1}$ that intersects $W$ in two almost vertical analytic disks.

Proposition 4 (Graph transform). For any $c \in \mathcal{C}$, there exists $K>1$ and $\varepsilon>0$ such that for any $a, 0<|a|<\varepsilon$, we have: for any analytic disk $\mathbb{D} \subset W$ verifying

(1) $\mathbb{D}=\left\{\left(\begin{array}{c}\phi(y) \\ y\end{array}\right), y \in \mathbb{D}_{R}\right\}$ where $\phi$ is analytic,

(2) $T_{w} \mathbb{D} \subset C_{K}(w)$ for any $w \in \mathbb{D}$,

then $H_{a, c}^{-1}(\mathbb{D}) \cap W$ is the union of two disks with the same properties.

Proof. We leave this result to the reader, who can use the implicit function theorem and then Rouché's theorem.

Lemma 9. When a tends to zero, the almost vertical analytic disks of the foliation become vertical.

Proof. For $n \geqslant 0$ we set $H_{a, c}^{\circ n}\left(\begin{array}{l}x \\ y\end{array}\right)=\left(\begin{array}{l}h_{1}^{n}(a, x, y) \\ h_{2}^{n}(a, x, y)\end{array}\right)$. Let $\left(\begin{array}{c}\psi(a, y) \\ y\end{array}\right)$ be a graph mapped by $H_{a, c}^{\circ n}$ into the graph $\left(\begin{array}{c}\phi(a, y) \\ y\end{array}\right) \subset U_{0}-U_{1}$. Then $h_{1}^{n}(\psi(a, y), y)=\phi\left(a, h_{2}^{n}(a, x, y)\right)$. When $a=0, \phi(0,)=$. const., and $h_{1}^{n}(0, x, y)=P_{c}^{\circ n}(x)$. Thus when $a=0$, $P_{c}^{\circ n}(\psi(0, y))=$ const. We take then the derivative and we obtain

$$
\left(P_{c}^{\circ n}\right)^{\prime}(\psi(0, y)) \cdot \frac{\partial}{\partial y} \psi(0, y)=0 .
$$

But the first $n$ iterates of $\psi(0, y)$ stay in $\mathcal{U}_{0}$, which does not contain the critical point 0 of $P_{c}$. Hence $\frac{\partial}{\partial y} \psi(0, y)=0$.

At this point with the help of holomorphic motions, we know that $K_{a, c}^{+} \cap W$ is homeomorphic to $\left(K_{c} \cap A(c)\right) \times \mathbb{D}_{R}$. 
3.2.2. Second half: $\boldsymbol{W} \cap \boldsymbol{U}^{+}$. It is easier to work in $U^{+}$because of the existence of the function $G^{+}$measuring the rate of escape to infinity.

Proposition 5 (Hubbard, Oberste-Vorth). The limit

$$
G^{+}=\lim _{n \rightarrow \infty} \frac{1}{2^{n}} \log _{+}\left\|H_{a, c}^{\circ n}\left(\begin{array}{l}
x \\
y
\end{array}\right)\right\|
$$

exists, is continuous on $\mathbb{C}^{2}$, pluri-harmonic on $U^{+}$, and satisfies the functional equation $G^{+}\left(H_{a, c}\left(\begin{array}{l}x \\ y\end{array}\right)\right)=2 G^{+}\left(\begin{array}{l}x \\ y\end{array}\right)$. Moreover, $U^{+}$is given by $U^{+}=\left\{\left(\begin{array}{l}x \\ y\end{array}\right) \mid G^{+}\left(\begin{array}{l}x \\ y\end{array}\right)>0\right\}$.

With this function we can straighten $U_{R}^{+}=U^{+} \cap\{|y|<R\}$ :

Lemma 10 (Coordinates on $U_{R}^{+}$). For any $(a, c)$ as in the main theorem, the open set $U_{R}^{+}$is biholomorphic to the domain $(|x|>1,|y|<R)$.

Proof. This is lemma 3.26 of [10]. In this article Fornæss and Sibony prove the following results.

- For any $y \in \mathbb{D}_{R}$ the function $x \mapsto G^{+}(x, y, a, c)$ has an harmonic conjugate $x \mapsto H(x, y, a, c)$ defined only modulo $2 \pi$.

- The map $F(x, y, a, c)=\exp \left(G^{+}(x, y, a, c)+i H(x, y, a, c)\right)$ is well defined.

- The map $\Theta(x, y, a, c)=(F(x, y, a, c), y)$, is the biholomorphism we are looking for. In addition it depends analytically on the parameters.

The next lemma is almost straightforward. Let us write $\mathbb{T}_{R}$ the solid torus $\mathbb{S}^{1} \times \mathbb{D}_{R}$.

Lemma 11. For any $(a, c)$ as in the main theorem, if we set

$$
\Omega=\left(0<G^{+}(x, y)<\ln 2\right) \cap(|y|<R)
$$

and $\Omega^{\prime}=J_{R}^{+} \cup \Omega$, then there exists a homeomorphism

$$
\begin{aligned}
\Phi_{U^{+}}: \Omega^{\prime} & \longrightarrow\{1 \leqslant|\zeta|<2\} \times \mathbb{T}_{R} \\
(x, y) & \longmapsto(r,(s, z))
\end{aligned}
$$

satisfying: if $\left(x^{\prime}, y^{\prime}\right),(x, y) \in \Omega^{\prime}$ are such that $\left(x^{\prime}, y^{\prime}\right)=H_{a, c}(x, y)$ then $r^{\prime}=r^{2}$.

Proof. This is simply a matter of rewriting the map $F(x, y, a, c)$ in polar coordinates $(r, \theta)$. One has

$$
(F(x, y, a, c), y)=(r,(\theta, y)) \in\{1<|\zeta|<2\} \times \mathbb{T}_{R} .
$$

Thus the $r$-coordinate is just $\exp \left(G^{+}\right)$. But remember we can use the functional equation $G^{+}\left(H_{a, c}\left(\begin{array}{l}x \\ y\end{array}\right)\right)=2 G^{+}\left(\begin{array}{l}x \\ y\end{array}\right)$. This proves the lemma at least in $\Omega$. Now Fornæss and Sibony make the following remark. 
Remark 3 (Fornæss-Sibony). If we set

$$
\Theta^{-1}(\zeta, y, a, c)=(\Gamma(\zeta, y, a, c), y)
$$

then for fixed $\zeta$, we get a leaf of the foliation of $\Omega$. But the $\lambda$-lemma of ManeSad-Sullivan (see the appendix) allows us to extend this foliation to a foliation of $\bar{\Omega}$. Hurwitz's lemma implies that this foliation of $J_{R}^{+}$coincides with the previous one.

Thus we can extend the homeomorphism to $\Omega^{\prime}=J_{R}^{+} \cup \Omega$. In the whole solid torus $J_{R}^{+}$one has $r=\exp (0)=1$ and of course the fact that $H_{a, c}\left(J_{R}^{+}\right) \subset J_{R}^{+}$is related to the equation $1^{2}=1$ !

This completes the proof of the lemma.

Remark 4. There is also a more dynamical way of obtaining the foliation by disks. Indeed, near the infinity in $U^{+}$there exists a holomorphic function $\phi^{+}$satisfying the functional equation $\phi^{+} \circ H_{a, c}=\left(\phi^{+}\right)^{2}$. In our case, we just said that this function can be analytically continued in the whole domain $U^{+} \cap\{|y| \leqslant R\}$.

The naturality of our construction is supported by the fact that the following very useful theorem comes immediately as an easy consequence.

Theorem 4 (Semi-conjugacy with $P_{c}$ ). There exists a continuous map

$$
\pi: W \rightarrow A(c)
$$

such that if we set $A^{\prime}(c)=P_{c}^{-1}(A(c))$ and $W^{\prime}=W \cap H^{-1}(W)$ then the following diagram commutes:

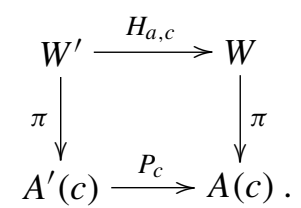

We call radial cylinder of W each set $\pi^{-1}(I)$, where I is a radial segment of $A(c)$.

Proof. The projection is given by the following recipe: for any point in $W$, take the almost vertical disk $x=\phi(a, y)$ through it, make $a=0$ and project this disk vertically in $A(c)$, or if one prefers, set $\pi(x, y)=\phi(0, y)$.

Let $\left(x^{\prime}, y^{\prime}\right),(x, y)$ be two points in $W$ such that:

- $\left(x^{\prime}, y^{\prime}\right)=H_{a, c}(x, y)$;

- $x^{\prime}=\phi\left(a, y^{\prime}\right)$ and $x=\phi(a, y)$. 
Then necessarily $\phi^{\prime}\left(a, y^{\prime}\right)=\phi^{2}(a, y)+c-a y$. So if we make $a=0$ we obtain $\phi^{\prime}\left(0, y^{\prime}\right)=P_{c}(\phi(0, y))$.

\section{Remark 5.}

(1) Since $P_{c}$ is conjugate to $z \mapsto z^{2}$ in the annulus $A(c)$, we also have a semiconjugacy to $z \mapsto z^{2}$. We knew that already in $W \cap U^{+}$because of the existence of $\phi^{+}$.

(2) There is a similar semi-conjugacy in the proposition 5.3 of [16] but since the authors do not use holomorphic motions they need to prove that the fibers are almost vertical by difficult ways involving some differential equations.

3.2.3. End of the proof of the thickening proposition. This is simply a matter of putting back together one half with the other, each one being foliated by disks parametrized by $y \in \mathbb{D}_{R}$.

3.3. Adapted coordinates on $\boldsymbol{W}$. In $W$ we constructed the analog of the level sets ( $r=$ const.) from the model. It remains to construct in $W$ the analog of the radial segments $(\theta=$ const. $)$

\subsubsection{Angular part. We want to show}

Proposition 6. There exists a homeomorphism $h^{\prime}:[1 / 2,2] \times \mathbb{T} \rightarrow W$ satisfying: If $\left(x^{\prime}, y^{\prime}\right)=h^{\prime}\left(r^{\prime}, \theta^{\prime}\right)$, and $(x, y)=h^{\prime}(r, \theta)$ are such that

$$
\left(x^{\prime}, y^{\prime}\right)=H_{a, c}(x, y) \text {. }
$$

then necessarily $r^{\prime}=r^{2}$.

Proof. We already know one half of this proposition: the one concerning $\left(J^{+} \cup U^{+}\right) \cap$ $\{|y|<R\}$. For the other one, $K_{R}^{+}-B$, we already have a foliation by solid tori, each one cutting $\{y=0\}$ along a circle obtained by a holomorphic motion of a circle in the annulus $A(c)$. The whole annulus $A(c)$ can be straightened into the annulus $A=\left\{\frac{1}{2}<|z|<2\right\}$, so that we can now parametrize the tori by $t \in[1 / 2,1]$. Since the Hénon map sends a torus in $W$ into another one, we have defined a monotone map $s$ from $[1 / 2,1]$ to itself. It is easy to conjugate it to $t \mapsto t^{2}$ by the following recipe: send the segment $[1 / 2, s(1 / 2)]$ on $[1 / 2,1 / \sqrt{2}]$ by a linear map $L$ and then send each point $t^{\prime}=s^{\circ n}(t)$ on $(L(t))^{\frac{1}{2^{n}}}$. 


\subsubsection{Radial part}

Outline of the proof. We will divide our proof into several steps:

(1) foliate $W-H_{a, c}(W)$ by radial segments;

(2) extend the foliation by forward iteration to $W-\bigcap_{n \geqslant 1} H_{a, c}^{\circ n}(W)$;

(3) use a continuity argument, in order to extend it to the whole $W$.

First step: foliation of $\boldsymbol{W}-\boldsymbol{H}_{\boldsymbol{a}, \boldsymbol{c}}(\boldsymbol{W})$. The topology of $W-H_{a, c}(W)$ is given by this lemma:

Lemma 12. The space $\bar{W}-\operatorname{int}\left(H_{a, c}(W)\right)$ is a locally trivial fiber bundle with base the annulus $A(c)$ and with fiber the Riemann sphere with three open disjoint disks removed.

Proof. This can be seen with the help of the semi-conjugacy $\pi: W \rightarrow A(c)$ between $H_{a, c}$ and $P_{c}$ : indeed we know that $H_{a, c}$ maps the two disks $\pi^{-1}(x)$ and $\pi^{-1}(-x)$ into the bigger disk $\pi^{-1}\left(P_{c}(x)\right)$.

A similar statement concerning the larger sets $W-H_{a, c}^{\circ n}(W)$ is immediate. Since there is always a section of such a fiber bundle over a radial segment of the annulus $A(c)$, we also have

Corollary 1. For any radial cylinder $C$, and for any $n \geqslant 1$ the intersection $C \cap H_{a, c}^{\circ n}(W)$ is made of $2^{n}$ disjoint cylinders.

Note that the horizontal boundary of $W$ is foliated by the horizontal annuli $W \cap$ $\left\{y=R e^{i \theta}\right\}$. Each such annulus is mapped by $H_{a, c}$ into an almost horizontal double cover of the annulus and these images foliate the boundary of $H_{a, c}(W)$. Thus there exists a natural foliation of the horizontal boundary $\partial_{\mathrm{hor}}\left(\bar{W}-H_{a, c}(W)\right)$ by onedimensional complex manifolds.

Proposition 7 (Extension of the natural foliation). There exists a homeomorphism

$$
\Upsilon:\left(J_{c} \times \mathbb{D}-f_{p}\left(J_{c} \times \mathbb{D}\right)\right) \times[1 / 2,2] \rightarrow W-H_{a, c}(W)
$$

satisfying

(1) $\Upsilon$ maps $\left(J_{c} \times \mathbb{D}-f_{p}\left(J_{c} \times \mathbb{D}\right)\right) \times\{1\}$ on $J_{R}^{+}-H_{a, c}\left(J_{R}^{+}\right)$;

(2) the foliation by the segments $\Upsilon(\{z\} \times[1 / 2,2])$ where $z \in \partial\left(J_{c} \times \mathbb{D}-f_{p}\left(J_{c} \times \mathbb{D}\right)\right)$ coincides with the natural foliation on the horizontal boundary of $W-H_{a, c}(W)$. 
Proof. We break the proof into two lemmas: first we remove a radial cylinder and we find a foliation, and then we adjust the construction along the removed cylinder.

Lemma 13 (Foliation above $A(c)$ with a slit). In $\bar{W}-\operatorname{int}\left(H_{a, c}(W)\right)$ with a radial cylinder $C$ removed, there exists a foliation by radial segments that agrees with the natural foliation on the horizontal boundary of $\bar{W}-\operatorname{int}\left(H_{a, c}(W)\right)$.

Proof. Let us represent the annulus $A(c)$ as the image by the exponential map of $[1 / 2,2] \times[0,2 \pi$. Then we can consider a topological disk $\Delta=[1 / 2,2] \times$ ] $-\varepsilon, 2 \pi+\varepsilon$ [ whose image by the exponential covers $A(c)$. Let us call $\widetilde{A}(c)$ the annulus $A(c)$ with a slit that corresponds to the segment $[1 / 2,2] \times\{0\}$.

Pick a basepoint $z_{0}$ in $\Delta$ and call $X$ the fiber of $z_{0}$, which is a closed disk with two smaller open disks removed. Through any point of $\partial X$ there is a unique leaf of the natural foliation. By moving the point $z \in \Delta$ and following each point of $\partial X$ as it slides along its own leaf, we define a holomorphic motion of $X$ parametrized by the disk $\Delta$. Therefore Słodkowski's theorem (see the appendix) can be applied and gives a holomorphic motion of the whole set $X$ (indeed of the whole complex plane), parametrized by the same disk $\Delta$. By doing this we can fill in the space between $W$ and $H_{a, c}(W)$ by a family of graphs of holomorphic functions on $\widetilde{A}(c)$. Any such disk is almost horizontal: indeed the holomorphic motion of a point inside $X$ cannot cross the exterior circle for injectivity reasons, so the derivative of the function is controlled through the Cauchy formulas. Therefore every such disk is transverse to any almost vertical disk $\pi^{-1}(x)$. By intersecting with a radial cylinder, one can obtain the desired radial segments.

Lemma 14 (Adjusting the monodromy). Let $C$ be the radial cylinder removed from $\bar{W}-\operatorname{int}\left(H_{a, c}(W)\right)$, and let

$$
u:[0,2 \pi] \times([1 / 2,2] \times X) \rightarrow \overline{W-(H(W) \cup C)}
$$

be the trivialization obtained by the holomorphic motion. Then there exists a homeomorphism s from $[0,2 \pi] \times[1 / 2,2] \times X$ to itself such that the restrictions of $u \circ s$ on $\{0\} \times[1 / 2,2] \times X$ and $\{2 \pi\} \times[1 / 2,2] \times X$ agree.

Proof. Since we used a motion on a topological disk covering $A(c)$, we have two different sets of radial segments on the cylinder $C$, depending on which side we approach the removed cylinder. These foliations coincide on the boundary of $C-H_{a, c}(W)$, but not inside a priori. The trivialization $u$ induces a monodromy map

$$
\mu:\left(u_{\mid\{2 \pi\} \times([1 / 2,2] \times X)}\right)^{-1} \circ u_{\mid\{0\} \times([1 / 2,2] \times X)}
$$

that needs to be adjusted. Let us take $w \in C$ such that $w=u(0,1 / 2, z)=$ $u\left(2 \pi, 1 / 2, z^{\prime}\right)$. A priori we have two segments:

$$
u(\{0\} \times[1 / 2,2] \times\{z\}) \text { and } u\left(\{2 \pi\} \times[1 / 2,2] \times\left\{z^{\prime}\right\}\right) .
$$


The fact that they do not coincide (except at $w$ ) gives us a family $h_{r}, r \in[1 / 2,2]$ of homeomorphisms of

$$
X=\overline{\mathbb{D}}_{0}-\left(\stackrel{\circ}{\mathbb{D}}_{1} \cup \stackrel{\circ}{\mathbb{D}}_{2}\right)
$$

that are the identity on the boundary. Moreover, by construction, we know that $h_{1 / 2}=$ Id. In order to sew back along the cylinder we just have to reparametrize this family by $[0,2 \pi]$ and to replace $u$ by $v=u \circ s$, where $s$ is defined by

$$
\begin{aligned}
s:[0,2 \pi] \times[1 / 2,2] \times X & \longrightarrow[0,2 \pi] \times[1 / 2,2] \times X \\
(\theta, r, z) & \longmapsto\left(\theta, r, h_{\theta}(z)\right) .
\end{aligned}
$$

This finishes the proof of the proposition.

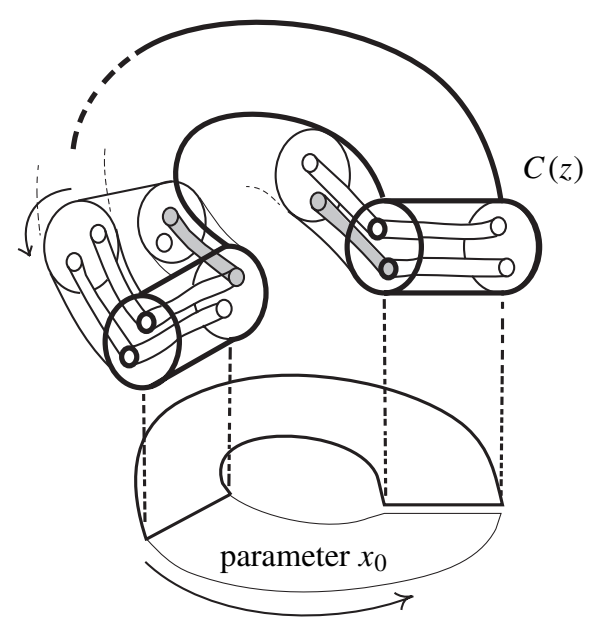

Figure 2. Using Slodkowski's theorem.

Second step: forward iteration. Let us work in a single radial cylinder. We extend the foliation by the dynamics: in order to fill in $\bar{C}(\zeta)-H_{a, c}^{\circ 2}(W)$ we look for the four preimages of $\zeta$ by $P_{c}^{2}$, then we fill in the corresponding cylinders and we map them back in $\bar{C}(\zeta)$. In this setting the notion of projective limit arises naturally.

Projective limit. For a complex polynomial $p$, we set $\hat{\mathbb{C}}_{p}=\underset{\lim }{\longleftarrow}(\mathbb{C}, p)$.

This gives a point and its history under the iteration by $p$ :

$$
\hat{\mathbb{C}}_{p}=\left\{\left(\ldots, z_{-2}, z_{-1}, z_{0}\right) \mid p\left(z_{-i-1}\right)=z_{-i} \text { for all } i=0,1,2, \ldots\right\} .
$$

There is an induced bijective map $\hat{p}: \hat{\mathbb{C}}_{p} \rightarrow \hat{\mathbb{C}}_{p}$ given by the shift

$$
\hat{p}\left(\ldots, z_{-2}, z_{-1}, z_{0}\right)=\left(\ldots, p\left(z_{-2}\right), p\left(z_{-1}\right), p\left(z_{0}\right)\right)=\left(\ldots, z_{-1}, z_{0}, p\left(z_{0}\right)\right) .
$$


We can restrict this construction to $\left(J_{c}, P_{c}\right)$ instead of $(\mathbb{C}, p)$. Let us write $\hat{J}_{c}$ this new projective limit.

Lemma 15. Let us recall that $\mathcal{U}_{n}=\left\{(x, y) \in W ; H_{a, c}^{\circ n}(x, y) \in W\right\}$ and set $\mathcal{V}_{n}=$ $H_{a, c}^{\circ n}\left(\mathcal{U}_{n}\right)$. Then

$$
\overline{\mathcal{V}_{n+1}} \cap W \subset \mathcal{V}_{n}, \quad \text { and } \bigcap_{n} \mathcal{V}_{n}=K^{-} \cap W .
$$

Proof. This is proposition 4.10 of [16] and also lemma 3.22 of [10].

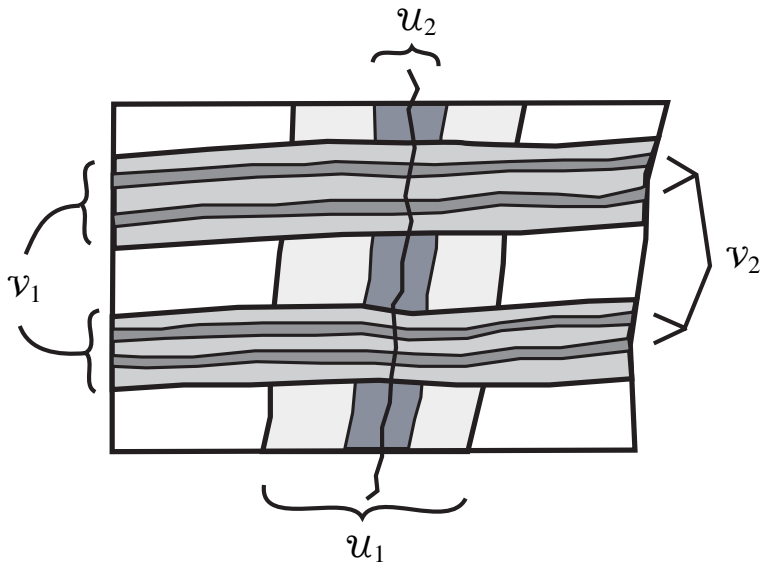

Figure 3. $\mathcal{V}_{i}$.

We can restrict this result to a given radial cylinder:

Lemma 16. For any cylinder $C(\zeta)$, if we write

$$
\hat{J}_{c}(\zeta)=\left\{\left(\ldots, \zeta_{-1}, \zeta_{0}\right) \in \hat{J}_{c} \mid \zeta_{0}=\zeta\right\}
$$

then

$$
K_{a, c}^{-} \cap C(\zeta)=\bigcup_{\hat{\zeta} \in \hat{J}_{c}(\zeta)} \bigcap_{n \geqslant 0} H_{a, c}^{\circ n}\left(C\left(\zeta_{-n}\right)\right) .
$$

Thus we still have to extend the foliation to $W \cap K^{-}$.

\section{Third step: extending to $W$}

Lemma 17. For any $\left(\ldots, \zeta_{-1}, \zeta_{0}\right) \in \hat{J}_{c}$, the family $\bigcap_{n=0}^{N} H_{a, c}^{\circ n}\left(C\left(\zeta_{-n}\right)\right)$ consists of a decreasing sequence of nested cylinders, whose intersection reduces to a segment parametrized by the radial segment $I\left(\zeta_{0}\right)$. 
Proof. It is enough to take an almost vertical disk $\mathbb{D}$ in the cylinder $\bar{C}$ and to show that the $\mathbb{D} \cap H_{a, c}^{\circ n}\left(C\left(\zeta_{-n}\right)\right)$ form a decreasing sequence of nested disks whose diameters tend to 0 .

But this convergence of the diameters to zero is a consequence of lemma 3.23 of [10]:

Lemma 18. For any $n \geqslant 0$ if we set, $V_{n}=H_{a, c}^{\circ n}\left(W_{n}\right)$, and for any $x_{0} \in A(c)$, $V_{n, x_{0}}=V_{n} \cap\left\{x=x_{0}\right\}$, then there exists a constant $C$ such that

$$
\operatorname{diam}\left(V_{n, x_{0}}\right) \leqslant C|a|^{n}
$$

End of the proof. At this point we know how to fill in any radial cylinder by radial segments that are preserved by $H_{a, c}$ and that are transverse to $J_{R}^{+}$. This ends the proof of the conjugacy theorem in $W$.

\section{Conjugacy in the basin of attraction}

Notations. The curve $\gamma_{0}$ is the inside boundary of the annulus $A(c)$ and at the same time it bounds a topological disk $\mathbb{D}(c)$ containing 0 and $c$. The bidisk $B=$ $\overline{\mathbb{D}(c)} \times \mathbb{D}_{R}$ is inside the basin of attraction of the attractive fixed point. If we set $K_{R}^{+}=K^{+} \cap\{|y|<R\}$ then we can consider a larger bidisk $\mathscr{B}=K_{R}^{+}-H_{a, c}^{-1}(W)$ whose vertical boundary is the set $\left\{p \in W \mid H_{a, c}(p) \in\left(\gamma_{0}\right) \times \mathbb{D}_{R}\right\}$.

Here is the goal of this section:

Theorem 5 (Conjugacy in int $\left(K^{+}\right)$). There exists a homeomorphism

$$
\Psi: \overline{\mathcal{B}}-\operatorname{int}\left(H_{a, c}(\mathscr{B})\right) \rightarrow[1 / 2,1 / \sqrt{2}] \times \mathbb{S}^{3},
$$

such that:

(1) $\Psi$ maps $\partial \mathscr{B}$ on $\left\{\frac{1}{\sqrt{2}}\right\} \times \mathbb{S}^{3}$ and $\partial\left(H_{a, c}(\mathscr{B})\right)$ on $\left\{\frac{1}{2}\right\} \times \mathbb{S}^{3}$;

(2) $\Psi$ conjugates $H_{a, c}$ with $g$ as a map from the outer boundary to the inner boundary;

(3) for any $p \in W$ such that $H_{a, c}(p) \in \bar{B}-\operatorname{int}\left(H_{a, c}(\mathscr{B})\right)$ we have

$$
\Psi \circ H_{a, c}(p)=g \circ \Theta(p) \quad \text { "consistency with the conjugacy on } W " .
$$




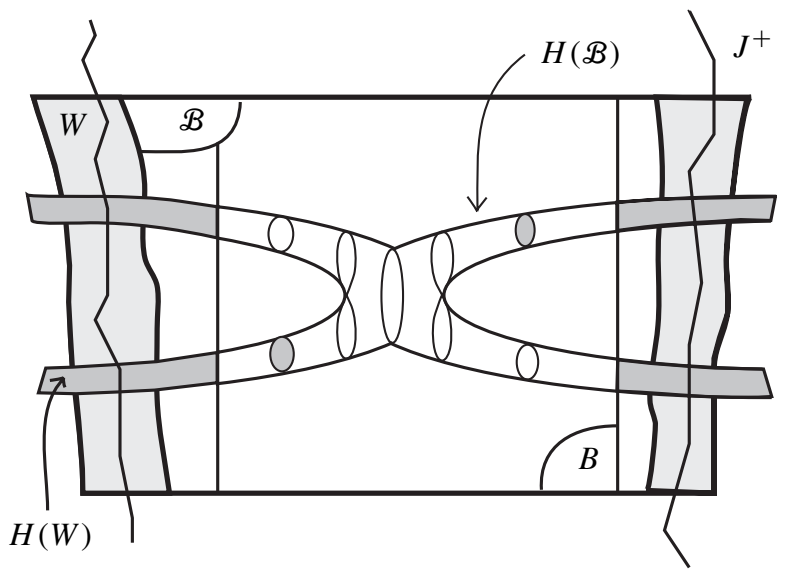

Figure 4. Filling in the basin of attraction.

4.1. First step. Let us write the 3 -sphere $\mathbb{S}^{3}$ as the union of two solid tori $\mathbb{T}_{0}, \mathbb{T}_{1}$. In the following proposition we fill in the space between $\mathscr{B}$ and its image $H_{a, c}(\mathscr{B})$ by a one-parameter family of 3 -spheres.

Proposition 8 (Family of 3-spheres). There exists a homeomorphism

$$
\lambda: \mathbb{S}^{3} \times[0,1] \rightarrow \overline{\mathcal{B}}-\operatorname{int}\left(H_{a, c}(\mathcal{B})\right),
$$

such that:

(1) $\lambda\left(\mathbb{S}^{3} \times\{0\}\right)=\partial \overline{\mathcal{B}}$;

(2) $\lambda\left(\mathbb{S}^{3} \times\{1\}\right)=\partial H_{a, c}(\overline{\mathcal{B}})$;

(3) $\lambda$ maps $\mathbb{T}_{0} \times[0,1]$ on $\left(H_{a, c}\left(\mathcal{U}_{1}\right)\right) \cap \bar{B}$ and satisfies the following conditions:

- the solid tori $\lambda\left(\mathbb{T}_{0} \times\{t\}\right)$ coincide with the solid tori arising in the foliation of $u_{1}$;

- the foliation by the segments $\lambda(\{\theta\} \times[0,1])$, where $\theta \in \mathbb{T}_{0}$ coincides with the foliation obtained in $W$.

\subsection{Proof of the Proposition 8}

Outline of the proof. Let us give an intuitive description of one 3-sphere belonging to our foliation. First imagine the horizontal boundary of a standard bidisk: it is a solid torus made of horizontal disks. Its boundary is a torus $T$ over the circle $\mathbb{S}$. Now 
the other solid torus that we will glue to $T$ is made of "vertical disks" that look like a truncated mountain with two peaks. Now when we follow the circle $\mathbb{S}$, the fiber turns, so that after one complete circle the two peaks are exchanged.

Now one can imagine that the time parametrizes our one-parameter family of spheres and acts by "eroding the mountains".

Our first lemma describes how $H(\mathscr{B})$ can be seen as a thick horizontal parabola in $\mathbb{C}^{2}$. When we intersect it with a vertical disk we get two disks, or a lemniscate or a single disk.

Lemma 19. The topology of $H(\mathscr{B})$ is described as follows:

(1) $H_{a, c}(\mathscr{B}) \cap\left\{x=x_{0}\right\}=\left\{\left(x_{0}, y\right) / y^{2}=a^{2}\left(x_{0}-c-a \cdot r e^{i \theta}\right), 0 \leqslant r \leqslant R, \theta \in \mathbb{R}\right\}$.

(2) There exists a vertical cylinder $\left\{0 \leqslant\left|x_{0}-c\right| \leqslant r^{\prime}\right\} \times \mathbb{C}$ outside of which $H_{a, c}(\mathscr{B}) \cap\left\{x=x_{0}\right\}$ is the union of two topological disks whose boundaries can be parametrized by $\arg \left(x-x_{1}\right)$ and $\arg \left(x-x_{2}\right)$, where

$$
\left\{x_{1}, x_{2}\right\}=\left\{x=x_{0}\right\} \cap\left\{\left(\begin{array}{c}
x^{2}+c \\
a x
\end{array}\right), x \in \mathbb{C}\right\} .
$$

Moreover, for a fixed parameter, the point we obtain depends continuously on $x_{0}$.

(3) If $\left|x_{0}-c\right|>r^{\prime}$, then for any $\alpha \in \mathbb{R}, H_{a, c}(\mathscr{B}) \cap\left\{x=e^{i \alpha} x_{0}\right\}$ is the image of $H_{a, c}(\mathscr{B}) \cap\left\{x=x_{0}\right\}$ by the rotation of center $O$ and of angle $\frac{\alpha}{2}$.

(4) There exists a real $r^{\prime \prime}>0$ such that if $\left|x_{0}-c\right|<r^{\prime \prime}$, then $\left\{x=x_{0}\right\} \cap H(\mathscr{B})$ is a topological disk.

Proof. We leave it to the reader. It is simply a matter of intersecting the parabola $H_{a, c}^{-1}\left\{x=x_{0}\right\}$ with the locus $\{|y|<R\}$.

\section{Radial cylinders in $\mathscr{B}$}

(1) in $\mathscr{B}-B$ the radial cylinders are defined as before: they are the intersections of the $C(\zeta)$ with $B-B$;

(2) by conformal representation the annulus $(B \cap\{y=0\})-\mathbb{D}\left(c, r^{\prime}\right)$ is foliated by radial segments: above each such segment we consider a straight radial cylinder made of genuine vertical disks;

(3) above each radial segment of the remaining disk $\mathbb{D}\left(c, r^{\prime}\right)$ we consider similarly the radial cylinder made of vertical disks.

Lemma 20. $H_{a, c}(\overline{\mathcal{B}}) \cap \overline{\mathbb{D}}\left(c, r^{\prime \prime}\right)$ is homeomorphic to a bidisk.

Proof. We leave it to the reader. 
Lemma 21 (Enlarging the topological bidisk). The space

$$
\left(\mathbb{D}\left(c, r^{\prime \prime}\right) \times \mathbb{D}_{R / 3}\right)-\left(H_{a, c}(\mathscr{B}) \cap\left(\mathbb{D}\left(c, r^{\prime \prime}\right) \times \mathbb{C}\right)\right)
$$

is homeomorphic to $\mathbb{D}\left(c, r^{\prime \prime}\right) \times\{1<|y|<2\}$.

Proof. This is just a matter of reparametrizing the annuli by a parameter of relative distance measured on half lines going through the origin.

Lemma 22 (Pants in $\left.\mathbb{R}^{3}\right)$. Let $C$ be a radial cylinder in $\mathcal{B}$ and $C^{\prime}=C \cap\left\{\left|x_{0}-c\right| \geqslant r^{\prime \prime}\right\}$. Then $\partial\left(H_{a, c}(\mathcal{B}) \cap C^{\prime}\right)$ is homeomorphic to a pair of pants $\Pi$ (that is, a Riemann sphere with three disjoint disks removed).

Proof. Left to the reader.

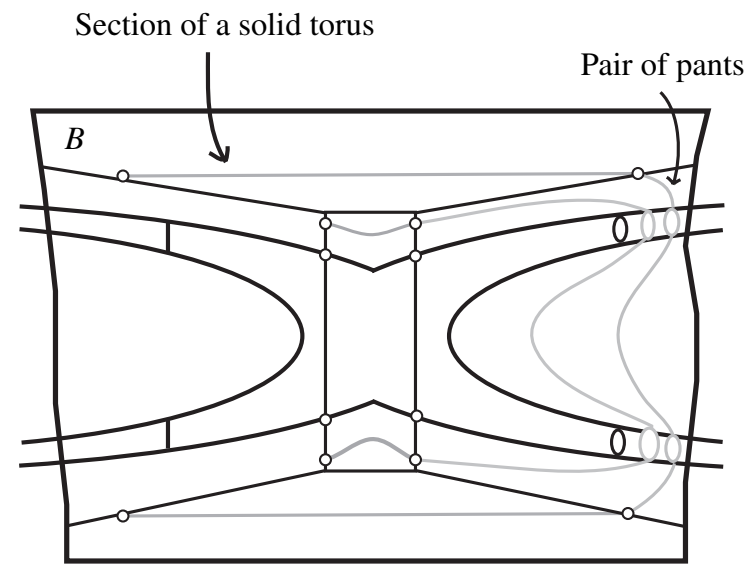

Figure 5. Family of pants.

Second step. "Taking off the pair of pants". Let us work now in a fixed radial cylinder. We need to give some notations.

(1) The bidisk $B=\mathbb{D}(c) \times \mathbb{D}_{R}$ is sitting inside the bidisk $\mathscr{B}$ whose horizontal boundary is given by $|y|=R$, and whose vertical boundary is the solid torus $W \cap H_{a, c}^{-1}\left(\partial_{\text {vert }} B\right)$.

(2) $B \cap\{y=0\}$ is the disk $\mathbb{D}(c)$, and $\mathscr{B} \cap\{y=0\}$ is a topological disk $\mathscr{D} \supset \mathbb{D}(c)$.

(3) The radial cylinder is written $C$ and its intersection with $\{y=0\}$ is a radial segment $L(t) \subset \mathbb{D}$ where $t \in[1,3]$, and $C(t)$ is the disk above the point $L(t)$. 
(4) For any $t \in[1,2], C(t)=\{L(t)\} \times \mathbb{D}_{R}$ (vertical disk), but for $t^{\prime} \in[2,3], C\left(t^{\prime}\right)$ is the almost vertical disk of the foliation of $W$.

(5) We assume that $L(1)$ is the closest to $c$ and sits on the circle $\left|x_{0}-c\right|=r^{\prime \prime}$.

(6) We call $C^{\prime}(t), t \in[1,3]$ the slightly deformed cylinder above the segment $L(t)$ whose width is an increasing linear function of $t$ so that $C^{\prime}(1)$ has radius $R / 3$ and $C^{\prime}(3)$ has radius $R$.

(7) We set $C([1,2])=C \cap \bar{B}$ and $C^{\prime}([1,2])=C^{\prime} \cap \bar{B}$, and also $C([2,3])=$ $C \cap \overline{\mathscr{B}}-\stackrel{\circ}{B}$ and $C^{\prime}([2,3])=C^{\prime} \cap \bar{B}-\stackrel{\circ}{B}$.

The following lemma describes the well-known action of "taking off a pair of pants".

Lemma 23. Let $\bar{\Pi}=\overline{\mathbb{D}}_{0}-\left(\stackrel{\circ}{\mathbb{D}}_{1} \cup \stackrel{\circ}{\mathbb{D}}_{2}\right)$ a pair of pants, then there exists a homeomorphism

$$
\chi: \bar{\Pi} \times[1,2] \rightarrow \overline{C^{\prime}}([1,2])-\operatorname{int}(H(\mathscr{B})),
$$

such that

(1) $\chi(\bar{\Pi} \times\{2\})=\overline{C^{\prime}(2)}-\operatorname{int}(H(B))$;

(2) $\chi(\bar{\Pi} \times\{1\})=\overline{C^{\prime}}([1,2]) \cap \partial(H(\mathscr{B}))$;

(3) for any $t \in[0,2]$ and any $z \in \partial \mathbb{D}_{1} \cup \partial \mathbb{D}_{2}$, we have $\chi(z, t)=\chi(z, 2)$;

(4) $\chi\left(\partial \mathbb{D}_{0} \times[1,2]\right)$ is the horizontal boundary of $C^{\prime}([1,2])$.

Proof. First we enlarge continuously the waist of the initial pair of pants so that it becomes a graph above a vertical disk. Its shape is that of a mountain with two flat peaks. Now we have a radial cylinder whose base is our graph and whose ceiling is the disk $C^{\prime}(2)$. It is easy to fill in the space between by a family of topological disks, each one having a straight circle as boundary.

Lemma 24. There exists a homeomorphism

$$
\omega:\left(\overline{\mathbb{D}}_{0}-\left(\stackrel{\circ}{\mathbb{D}}_{1} \cup \stackrel{\circ}{\mathbb{D}}_{2}\right)\right) \times[2,3] \rightarrow\left(\bar{C}^{\prime}([2,3])-\operatorname{int}\left(H_{a, c}(W)\right)\right)
$$

such that

(1) $\omega$ maps $\partial \mathbb{D}_{0} \times[2,3]$ in the horizontal boundary of $\bar{C}^{\prime}([2,3])$;

(2) for any $z \in\left(\partial \mathbb{D}_{1} \cup \partial \mathbb{D}_{2}\right)$, the segment $\omega(\{z\} \times[2,3])$ coincides with the segment belonging to the foliation of $W$.

Proof. It is enough to realize that in both cases we have a trivial fiber bundle with fiber a pair of pants. 
Then we put together these two lemmas. By doing the construction in all the radial cylinders, we obtain a one-parameter family of solid tori. The boundary of each one is a torus made of vertical circles. Now we can glue on each such torus a solid torus made of horizontal disks, in the same way the boundary of a complex bidisk is made of two solid tori glued along a torus. This ends the proof of the proposition.

4.3. End of the proof of the theorem. Let us summarize the situation. We proved that $\overline{\mathcal{B}}-\stackrel{\circ}{B}$ is a spherical shell homeomorphic to $\mathbb{S}^{3} \times[0,1]$. The conjugacy has been defined on the "exterior sphere" $\mathbb{S}^{3} \times\{1\}$ and also on $H_{a, c}(W)$ which intersects the shell on a set homeomorphic to $\mathbb{T} \times[0,1]$. It remains to define the conjugacy on the complement, which is also homeomorphic to $\mathbb{T} \times[0,1]$. On the torus $\mathbb{T} \times\{0\}$ two different sets of coordinates coexist: a priori the coordinates given by the homeomorphism with $\mathbb{S}^{3} \times[0,1]$ do not respect the conjugacy. The next lemma shows how one can deform continuously one system of coordinates into the other one.

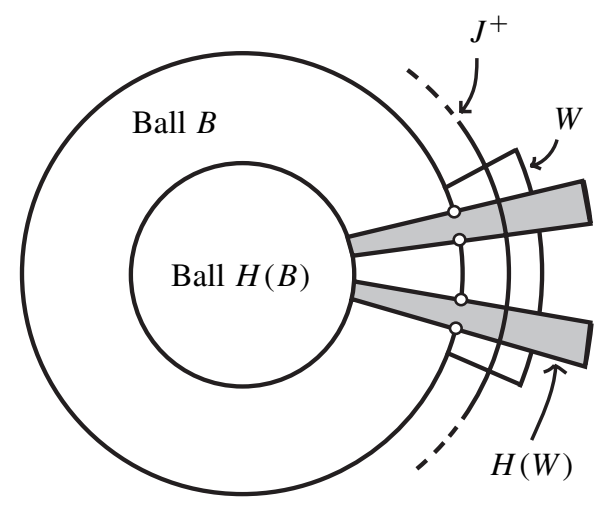

Figure 6. Filling in the spherical shell.

If we put coordinates $(z, t) \in \mathbb{T} \times[0,1]$ on $\overline{\mathcal{B}}-(\stackrel{\circ}{B} \cup \overbrace{H_{a, c}\left(\mathcal{U}_{1}\right)}^{\circ})$ we are led to the following situation:

Lemma 25. Let

$$
X=\mathbb{T} \times[0,1] \text { and } A \subset \mathbb{T} \times\{0\}
$$

be such that there exists two homeomorphisms $F_{1}, F_{2}$ of $A$ on $\mathbb{T} \times\{1\}$ defined by

$$
F_{1}(x, 0)=\left(f_{1}(x), 1\right) \text { and } F_{2}(x, 0)=\left(f_{2}(x), 1\right) .
$$

Then there exists a homeomorphism $\Delta: X \rightarrow X$ satisfying:

(1) $\Delta$ is the identity on $\partial \mathbb{T} \times[0,1]$ and on $\mathbb{T} \times\{0\}$; 
(2) $\Delta$ conjugates $F_{1}$ and $F_{2}$ on $A$.

This lemma is an immediate consequence of the following result, that will be proved in the next section:

Lemma 26. The space Homeo(T rel $\partial$ ) of all homeomorphisms from the solid torus to itself that are the identity on the boundary has the homotopy type of a point.

Proof of Lemma 25. Indeed, with Lemma 26 we have in Homeo(T rel d) a continuous path $g_{t}, t \in[0,1]$ joining the identity to $f_{2} \circ f_{1}^{-1}$. Then we set

$$
\Delta(z, t)=\left(g_{t}(z), t\right) .
$$

This $\Delta$ is the identity on $\partial \mathbb{T} \times[0,1]$ and $\mathbb{T} \times\{0\}$, and satisfies for any $(x, 0) \in A$ :

$$
\begin{aligned}
\Delta \circ F_{1}(x, 0) & =\Delta\left(f_{1}(x), 1\right) \\
& =\left(g_{1} \circ f_{1}(x), 1\right) \\
& =F_{2}(x, 0) \\
& =F_{2} \circ \Delta(x, 0) .
\end{aligned}
$$

This lemma ends the proof of the theorem.

\section{A topological lemma}

Lemma 27 (Homeo(T rel $\partial) \simeq *$ ). The space of homeomorphisms from $\mathbb{T}$ to itself that are the identity on the boundary has the homotopy type of a point.

Proof. This lemma is a consequence of two theorems. The first one is Smale's conjecture Diff $\left(\mathbb{S}^{3}\right) \simeq \mathrm{O}(4)$, proved by Allen Hatcher in [12]:

Theorem 6 (Smale's conjecture). The inclusion of the orthogonal group $\mathrm{O}(4)$ in $\operatorname{Diff}\left(\mathbb{S}^{3}\right)$ is a homotopy equivalence. Moreover Smale's conjecture is equivalent to:

(1) $\operatorname{Diff}\left(\mathbb{S}^{1} \times \mathbb{D}^{2}\right.$ rel $\left.\partial\right) \simeq *$,

(2) $\operatorname{Diff}\left(\mathbb{D}^{3} \operatorname{rel} \partial \mathbb{D}^{3}\right) \simeq *$.

The second theorem is due to J. Cerf in [6]:

Theorem 7. For any 3-dimensional manifold $M^{3}$,

$$
\operatorname{Diff}\left(\mathbb{D}^{3} \operatorname{rel} \partial \mathbb{D}^{3}\right) \simeq * \Longrightarrow \operatorname{Diff}\left(M^{3} \operatorname{rel} \partial M^{3}\right) \simeq \operatorname{TOP}\left(M^{3} \operatorname{rel} \partial M^{3}\right) .
$$

In this theorem one can also replace the category TOP by the piecewise-linear category PL. 
Remark 6. We might as well construct a path of diffeomorphisms by hand, without using the full strength of Hatcher's theorem, as pointed out by Ryan Budney. We also thank Professor Laudenbach for his help on 3-dimensional topology.

\section{Conjugacy in the escaping set}

Let us define

$$
V^{+}\left(\left[r,+\infty[)=\left\{\left(\begin{array}{l}
x \\
y
\end{array}\right) \in V^{+} \mid G^{+}\left(\begin{array}{l}
x \\
y
\end{array}\right) \geqslant \log r\right\} .\right.\right.
$$

Then our goal in this section is this theorem:

Theorem 8 (Conjugacy in $V^{+}$). Let us assume that the vertical boundary of $W$ is determined in $U^{+}$by the level set $\left\{G^{+}=2 \log s\right\}$. Then there exists a homeomorphism

$$
\Lambda: \mathbb{T} \times\left[s^{2},+\infty\left[\rightarrow V ^ { + } \left(\left[s^{2}, \infty[)\right.\right.\right.\right.
$$

satisfying

(1) $\Lambda$ conjugates $H_{a, c}$ with $g$;

(2) for any $r^{\prime} \geqslant s^{2}$ and any $z \in \mathbb{T}, G^{+}\left(\Lambda\left(z, r^{\prime}\right)\right)=\log r^{\prime}$;

(3) let $p$ be in $U^{+} \cap W$ such that $\Theta^{-1}(p)=(r, \theta)$ and let $N$ be in $\mathbb{N}$ such that $H_{a, c}^{\circ N}(p) \in V^{+}\left(\left[s^{2}, \infty[)\right.\right.$, then $g^{\circ N}(r, \theta)=\Lambda \circ H_{a, c}^{\circ N}(p)$ (compatibility with the trivialization of $W)$.

Remark 7. In the construction of $W$, the choice of the vertical boundary was free: the only condition needed was that $H_{a, c}(W)$ should intersect $W$ only in its vertical boundary ("transversality condition").

From now on we define $W \cap U^{+}$as follows: for fixed $R>0, U^{+} \cap \partial_{\text {vert }}(W)$ is the solid torus $\{|y| \leqslant R\} \cap\left\{\left|\phi^{+}\right|=R /|a|\right\}$. We let the reader verify that such a choice satisfies the transversality condition.

5.1. Using $\phi^{+}$as a coordinate. Here is a lemma describing the behaviour of $\phi^{+}$:

Lemma 28. When $\left\|\left(\begin{array}{l}x \\ y\end{array}\right)\right\|$ tends to $+\infty$ within $V^{+}$

$$
\phi^{+}(a, x, y)=x+\frac{a}{2} \frac{y}{x}+O\left(\frac{1}{|x|}\right),
$$

where $O\left(\frac{1}{|x|}\right)$ stands for a function $g(a, x, y)$ that satisfies: there exists $M>0$ and $r>0$ such that for any $a \in \mathbb{D}_{\varepsilon}^{*}$ and any $\left(\begin{array}{l}x \\ y\end{array}\right) \in V^{+}([r,+\infty[)$ we have $|x \cdot g(a, x, y)| \leqslant M$. 


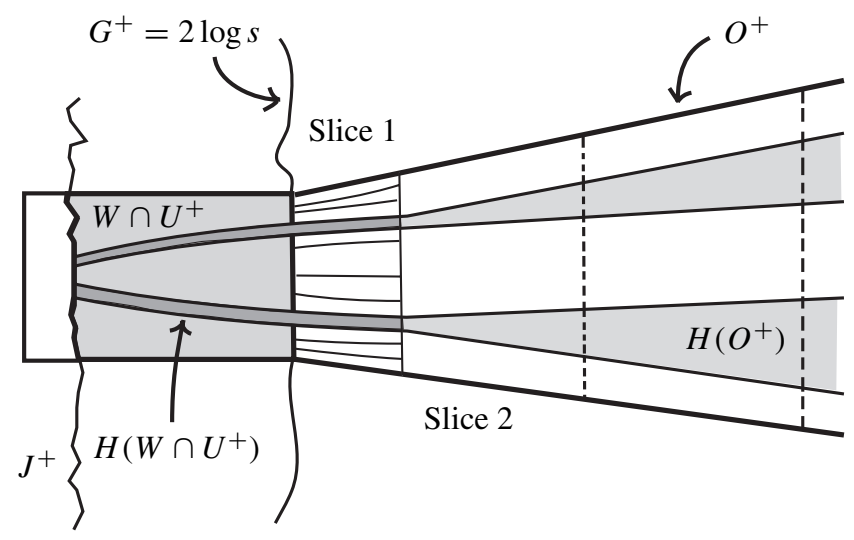

Figure 7. Coordinates in $\mathrm{O}^{+}$.

Proof. When $\left\|\left(\begin{array}{l}x \\ y\end{array}\right)\right\|$ tends to $+\infty$ in $V^{+}$, then $\frac{x^{2}+c+a y}{x^{2}}$ tends to 1 uniformly in $a \in \mathbb{D}_{\varepsilon}$. We can then set as in [24]

$$
x^{2}+c+a y=x^{2} e^{\beta(a, x, y)}
$$

where the function $\beta$ is bounded. Thus for $z=(a, x, y)$ the series

$$
\gamma(a, x, y)=\frac{1}{2} \beta(z)+\frac{1}{2^{2}} \beta\left(H_{a, c}(z)\right)+\cdots
$$

converges uniformly, therefore one can write $\phi^{+}(a, x, y)=x e^{\gamma(z)}$. In $\gamma(z)$ only the first term is leading, so one has $\gamma(z)=\frac{a}{2} \frac{y}{x^{2}}+O\left(\frac{1}{\left|x^{2}\right|}\right)$, where $O$ must be understood as: uniformly with respect to $a \in \mathbb{D}_{\varepsilon}$.

The next lemma introduces the set corresponding to $\mathbb{T} \times\left[s^{2},+\infty[\right.$ in the model: it is the result of a small modification of $V^{+}$, that is required if one wants to use $\phi^{+}$ as a coordinate.

Lemma 29. There exists $\beta>0$ small enough such that

$$
O^{+}=\left\{\left(\begin{array}{l}
x \\
y
\end{array}\right) \in V^{+}|| \phi^{+} \mid>\frac{R}{|a|(1-\beta)} \text { and } \frac{|y|}{\left|\phi^{+}\right|}<|a|(1-\beta)\right\}
$$

satisfies

(1) $\partial O^{+} \subset \stackrel{\circ}{V^{+}}$;

(2) $\bigcup_{n \geqslant 0} H_{a, c}^{\circ-n}\left(O^{+}\right)=U^{+}$;

(3) the mapping $\Phi=\left(\phi^{+}, y / \phi^{+}\right): O^{+} \rightarrow \mathbb{C}^{2}$ is a biholomorphism from $O^{+}$to $\left\{|\zeta|>\frac{R}{|a|(1-\beta)}\right\} \times \mathbb{D}_{|a|(1-\beta)}$ 
Proof. This is essentially a consequence of the fact that $\phi^{+} \sim x$ near the infinity, and that near the infinity $\left(\phi^{+}(a, x, y), \frac{y}{x}\right)$ is injective. This last fact is nothing else than lemma 6.3 of [15].

\subsection{Filling in the slices of $O^{+}$}

First slice of $\boldsymbol{O}^{+}$. From now on we set $s^{2}=\frac{R}{|a|(1-\beta)}$. We recall that the trivialization of $W$ is given by $\Theta:[1 / 2,2] \times \mathbb{T} \rightarrow W$. We change it a bit into a homeomorphism from $\left[1 / 2, s^{2}\right] \times \mathbb{T}$ into $W$ that we still call $\Theta$.

Proposition 9. There exists a homeomorphism

$$
T: \mathbb{T} \times\left[s^{2}, s^{4}\right] \rightarrow O^{+} \cap\left\{s^{2} \leqslant\left|\phi^{+}\right| \leqslant s^{4}\right\}
$$

satisfying

(1) $\Theta$ and $T$ coincide on $\mathbb{T} \times\left\{s^{2}\right\}$;

(2) for any $z \in \partial \mathbb{T}$, the segments $T\left(\{z\} \times\left[s^{2}, s^{4}\right]\right)$ coincide with the segments of the natural foliation of the boundary defined by the level sets $\left(\frac{y}{\phi^{+}}=\right.$const.);

(3) for any $z^{\prime} \in H_{a, c}\left(\partial\left(W \cap\left\{\left|\phi^{+}\right|=s\right\}\right)\right)$ the segments $T\left(\left\{z^{\prime}\right\} \times\left[s^{2}, s^{4}\right]\right)$ coincide with the images by $H_{a, c}$ of the segments belonging to the natural foliation of $\partial_{h} W$ defined by the level sets $\left(y=R e^{i \theta_{0}}\right)$.

Proof. Again we use holomorphic motions, in a simpler setting because we can use $\phi^{+}$ as a coordinate.

In the coordinates $\left(\phi^{+}, y / \phi^{+}\right)$, let $\mathbb{D}_{\zeta}$ be a vertical disk $\left\{\phi^{+}=\zeta\right\}$, with $\zeta \in$ $\left[s^{2}, s^{4}\right]$. Then the image by $H_{a, c}$ of the slice $W \cap\left\{s \leqslant\left|\phi^{+}\right| \leqslant s^{2}\right\}$ cuts two disks in $\mathbb{D}_{\zeta}$. Indeed we have the functional equation $\phi^{+} \circ H_{a, c}=\left(\phi^{+}\right)^{2}$. Thus,

$$
\mathbb{D}_{\zeta} \cap H_{a, c}\left(W \cap\left\{s \leqslant\left|\phi^{+}\right| \leqslant s^{2}\right\}\right)=\mathbb{D}_{\zeta} \cap\left(H\left(\mathbb{D}_{\zeta^{\prime}}\right) \cup H\left(\mathbb{D}_{-\zeta^{\prime}}\right)\right),
$$

where $\zeta^{\prime 2}=\zeta$. Let us call $X=\overline{\mathbb{D}_{0}}-\left(\stackrel{\circ}{\mathbb{D}}_{1} \cup \stackrel{\circ}{\mathbb{D}}_{2}\right)$ this disk with two smaller disks removed. The image by $H_{a, c}$ of each annulus $\left(y=R e^{i \theta_{0}}\right)$ in the slice $W \cap\{s \leqslant$ $\left.\left|\phi^{+}\right| \leqslant s^{2}\right\}$ is a double cover above the annulus $\left\{s^{2} \leqslant\left|\phi^{+}\right| \leqslant s^{4}\right\}$. By following an intersection of such a cover with a disk $\mathbb{D}_{\zeta}$ when $\zeta$ moves in the annulus with a slit parametrized by $\left\{\zeta=r e^{i \theta}, \theta \in\left[0,2 \pi\left[, r \in\left[s^{2}, s^{4}\right]\right\}\right.\right.$, we construct a holomorphic motion of $\partial X$ that we can extend to $\mathbb{C}$ by using Stodkowski's theorem. As for the case of $W$, we have to adjust the monodromy map in order to finish the proof.

Filling in the other slices. After the slice $O_{N}^{+}=O^{+}\left(\left[s^{2^{N}}, s^{2^{N+1}}\right]\right)$, we can apply the method above to the space $O_{N+1}^{+}-\operatorname{int}\left(H_{a, c}\left(O_{N}^{+}\right)\right)$. 
We do not have to worry about the interior of $H_{a, c}\left(O_{N}^{+}\right)$because it has the foliation induced by the foliation of $O_{N}^{+}$. By iterating this construction we can fill in all the slices, and this ends the proof of Theorem 8.

\section{Conclusion}

The proof of our main theorem is now finished: we just have to put everything together, the spherical shell in the basin of attraction, the tubular neighbourhood $W$, the open set $O^{+} \subset U^{+}$. The different trivializations agree, and by iterating forward and backward we can extend the conjugacy to $\mathbb{C}^{2}$.

\section{Remark 8.}

(1) Our proof should extend to the case where the Hénon map is of higher degree without too many difficulties.

(2) An interesting question would be to estimate the size of $\varepsilon$ in the main theorem.

\section{Appendix: Holomorphic motions}

We recall the basic notions about holomorphic motions.

Definition. If $X \subset \mathbb{C}$, and $\Lambda$ is a connected $\mathbb{C}$-analytic manifold with a base-point $\lambda_{0}$, then a holomorphic motion of $X$ parametrized by $\Lambda$ is a map $h: X \times \Lambda \rightarrow \overline{\mathbb{C}} \times \Lambda$ such that:

(1) $h\left(., \lambda_{0}\right): X \rightarrow \mathbb{C}$ is the canonical injection;

(2) $h$ is injective;

(3) $h(x,):. \Lambda \rightarrow \overline{\mathbb{C}}$ is $\mathbb{C}$-analytic for all $x \in X$.

Theorem 9 ( $\Lambda$-lemma, Mañe-Sad-Sullivan). Let $h: X \times \Lambda \rightarrow \overline{\mathbb{C}} \times \Lambda$ be a holomorphic motion of a subset $X$ of $\overline{\mathbb{C}}$. Then $h$ is continuous and has a continuous extension $\hat{h}: \bar{X} \times \Lambda \rightarrow \overline{\mathbb{C}} \times \Lambda$ which is a holomorphic motion of the closure $\bar{X}$ of $X$.

Theorem 10 (Słodkowski). Let $\mathbb{D}$ be the unit disk in $\mathbb{C}$, with $O$ the basepoint, and $X$ a subset of $\overline{\mathbb{C}}$. Any holomorphic motion of $X$ parametrized by $\mathbb{D}$ can be extended to a holomorphic motion of $\overline{\mathbb{C}}$ parametrized by $\mathbb{D}$.

For a proof, the reader can read [9] and also a new proof given by Chirka. 
Theorem 11 (Bers-Royden). Let $\Lambda$ be the open unit ball of a Banach space $E$ over $\mathbb{C}$. For any holomorphic motion $h$ of $X \subset \overline{\mathbb{C}}$ parametrized by $\Lambda$, there exists a holomorphic motion of $\overline{\mathbb{C}}$ parametrized by the ball of radius $1 / 3$ and coinciding with $h$ on $X$.

The proof of Bers-Royden's theorem given in [22] can be modified as follows.

Theorem 12 (Variant of Bers-Royden's theorem). Let $h$ be a holomorphic motion of $X \subset \overline{\mathbb{C}}$, parametrized by the bidisk $\Lambda=\mathbb{D}_{s} \times \mathbb{D}_{r}$. Let us assume that $h$ satisfies on $X$ the additional condition

$$
h(0, y, .)=\mathrm{Id} \text { for all } y \in \mathbb{D}_{r} .
$$

Then there exists a motion $\tilde{h}$ of $\overline{\mathbb{C}}$ parametrized by the bidisk $\mathbb{D}_{s / 3} \times \mathbb{D}_{r / 3}$ which coincides with $h$ on $X$ and also satisfies:

$$
\widetilde{h}(0, y, .)=\mathrm{Id} \text { for all } y \in \mathbb{D}_{r} .
$$

on the whole $\overline{\mathbb{C}}$

Proof. Let us assume for the beginning that $X \subset \mathbb{C}$ is a finite fixed set. There exists a natural map $\Phi$ from the space $\mathcal{M}\left(\mathbb{P}^{1}-X\right)$ of the Beltrami forms over $\mathbb{P}^{1}-X$ on the Teichmüller space $\mathcal{T}\left(\mathbb{P}^{1}-X\right)$, defined by integrating the Beltrami form:

$$
\Phi: \mu \rightarrow\left(\mathrm{Id}: X \rightarrow X_{\mu}\right)
$$

There exists a section $\Psi$ of this map, that is defined on the ball of radius $1 / 3$.

Now the holomorphic motion of $X$ defines a holomorphic path $Y_{\lambda}=\mathbb{P}^{1}-h_{\lambda}(X)$ in $\mathcal{T}\left(\mathbb{P}^{1}-X\right)$.

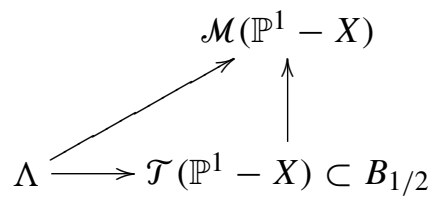

By Schwarz's lemma we know that the ball of radius $1 / 3$ is mapped into the ball of radius $1 / 2$. We can then compose by the section $\Psi$ in order to obtain a holomorphic family of Beltrami forms, thus giving us the desired holomorphic motion after integration of these forms. But now in the extended holomorphic motion, we have factored through the map $Y: \Lambda \rightarrow \mathcal{T}\left(\mathbb{P}^{1}-X\right)$. Therefore if $Y$ is constant along the disk $\lambda \cap(a=0)$, then the same thing is true for the extension.

For a general set $X$, we exhaust $X$ by an increasing family of finite subsets $\left(X_{n}\right)$ such that $X_{\infty}=\bigcup X_{n}$ is dense in $X$. 


\section{References}

[1] L. Bers, H. L. Royden, Holomorphic families of injections. Acta Math. 157 (1986), 259-286. Zbl 0619.30027 MR 0857675

[2] E. Bedford, J. Smillie, Polynomial diffeomorphisms of $\mathbb{C}^{2}$. VI: Connectivity of J. Ann. of Math. (2) 148 (1998), 695-735. Zbl 0916.58022 MR 1668567

[3] E. Bedford, J. Smillie, Polynomial diffeomorphisms of $\mathbf{C}^{2}$, VII: Hyperbolicity and external rays. Ann. Sci. École Norm. Sup. (4) 32 (1999), 455-497. Zbl 0952.37008 MR 1693587

[4] G. Buzzard, Extensions of Hénon maps to the closed 4-ball. Ergodic Theory Dynam. Systems 20 (2000),1319-1334. Zbl 0987.37034 MR 1786716

[5] L. Carleson, T. Gamelin, Complex dynamics. Universitext: Tracts Math., Springer-Verlag, New York 1993. Zbl 0782.30022 MR 1230383

[6] J. Cerf, Groupes d'automorphismes et groupes de difféomorphismes des variétés compactes de dimension trois. Bull. Soc. Math. France 87 (1959), 319-329. Zbl 0102.38703 MR 0116351

[7] J. Cerf, Sur les difféomorphismes de la sphère de dimension trois $\Gamma_{4}=0$. Lecture Notes in Math. 53, Springer-Verlag, Berlin . Zbl 0164.24502 MR 0229250

[8] A. Douady, Lecture notes for a graduate course. Orsay 2001.

[9] A. Douady, Prolongement de mouvements holomorphes (d'après Słodkowski et autres). Séminaire Bourbaki, Exposés 775-789, 1993/94; Astérisque 227 (1995), 7-20. Zbl 0832.30016 MR 1321641

[10] J. Fornæss, N. Sibony, Complex Hénon mappings in $\mathbf{C}^{2}$ and Fatou-Bieberbach domains. Duke Math. J. 65 (1992), 345-380. Zbl 0761.32015 MR 1150591

[11] J. Fornæss, N. Sibony, Complex dynamics in higher dimension, I. Complex Analytic Methods in Dynamical Systems (Rio de Janeiro, 1992); Astérisque 222 (1994), 201-231. Zbl 0813.58030 MR 1285389

[12] A. Hatcher, A proof of the Smale Conjecture $\operatorname{Diff}\left(S^{3}\right) \simeq \mathrm{O}(4)$. Ann. of Math. (2) 117 (1983), 553-607. Zbl 0531.57028 MR 0701256

[13] A. Hatcher, Notes on 3-dimensional topology. In preparation.

[14] A. Hatcher, Algebraic topology. Cambridge University Press, Cambridge 2002. Zbl 1044.55001 MR 1867354

[15] J. H. Hubbard, R. W. Oberste-Vorth, Hénon mappings in the complex domain, I: The global topology of dynamical space. Inst. Hautes Etudes Sci. Publ. Math. 79 (1994), 5-46. Zbl 0839.54029 MR 1307296

[16] J. H. Hubbard, R. W. Oberste-Vorth, Hénon mappings in the complex domain, II. In Projective and inductive limits of polynomials. Real and Complex Dynamical Systems (Hillerød, 1993). NATO Adv. Sci. Inst. Ser. C Math. Phys. Sci. 464, Kluwer, Dordrecht 1995, 89-132. Zbl 0874.54037 MR 1351520

[17] J. H. Hubbard, R. W. Oberste-Vorth, Linked Solenoid Mappings and the Non-Transversality Locus Invariant. Indiana Univ. Math. J. 50 (2001), 553-566. Zbl 1041.37008 MR 1857045

[18] J. H. Hubbard, P. Papadopol, V. Veselov, A Compactification of Hénon mappings in $\mathbf{C}^{2}$ as Dynamical Systems. Acta Math 184 (2000), 203-270. Zbl 0987.37035 MR 176811 
Vol. 81 (2006)

[19] J. H. Hubbard, Teichmüller Spaces and Applications to Topology, Geometry and Dynamics, Four theorems by W. Thurston. Matrix Editions. In preparation.

[20] J. H. Hubbard, The Hénon mapping in the complex domain. In Chaotic Dynamics and Fractals (Atlanta, GA, 1985), Notes Rep. Math. Sci. Engrg. 2, Academic Press, Orlando 1986, 101-111. Zbl 0601.32029 MR 0858009

[21] A. Katok, B. Hasselblatt, Introduction to the Modern Theory of Dynamical Systems. Encyclopedia Math. Appl. 54, Cambridge University Press Cambridge 1995. Zbl 0878.58020 MR 1326374

[22] C. McMullen, Riemann surfaces, dynamics and geometry. Course notes, Harvard University 2001.

[23] J. Milnor, Dynamics in one complex variable: introductory lectures. Preprint, Institute for Mathematical Sciences, SUNY, Stony Brook 1990.

[24] S. Morosawa, Y. Nishimura, M. Taniguchi, T. Ueda, Holomorphic dynamics. Cambridge Stud. Adv. Math. 66, Cambridge University Press, Cambridge 2000 Zbl 0979.37001 MR 1747010

[25] R. Remmert, Classical Topics in Complex Function Theory. Grad. Texts in Math. 172, Springer-Verlag, New York 1998. Zbl 0895.30001 MR 1483074

[26] Z. Słodkowski, Extensions of holomorphic motions. Ann. Scuola Norm. Sup. Cl. Sci. (4) 22 (1995), 185-210. Zbl 0835.30012 MR 1354904

Received June 7, 2005

Sylvain Bonnot, Institute for Mathematical Sciences, Stony Brook University, Stony Brook, NY 11794-3660, U.S.A.

E-mail: bonnot@math.sunysb.edu 